\title{
On Computation of Face Index of Certain Nanotubes
}

\author{
Ansheng Ye, ${ }^{1}$ Aisha Javed, ${ }^{2}$ Muhammad Kamran Jamil ${ }^{10},{ }^{3}$ Kanza Abdul Sattar, ${ }^{3}$ \\ Adnan Aslam $\left(\mathbb{1},{ }^{4}\right.$ Zahid Iqbal $\left(\mathbb{D},{ }^{5,6}\right.$ and Asfand Fahad $\mathbb{1}^{7}$ \\ ${ }^{1}$ School of Information Science and Engineering, Chengdu University, Chengdu 610106, China \\ ${ }^{2}$ Abdus Salam School of Mathematical Sciences, GC University, Lahore, Pakistan \\ ${ }^{3}$ Department of Mathematics, Riphah International University, 14 Ali Road, Lahore, Pakistan \\ ${ }^{4}$ Department of Natural Sciences and Humanities, University of Engineering and Technology, Lahore (RCET), Lahore, Pakistan \\ ${ }^{5}$ National University of Science and Technology, Islamabad, Pakistan \\ ${ }^{6}$ Department of Mathematics and Statistics, Institute of Southern Punjab, Multan, Pakistan \\ ${ }^{7}$ Department of Mathematics, COMSATS University Islamabad, Vehari Campus, Vehari 61110, Pakistan
}

Correspondence should be addressed to Adnan Aslam; adnanaslam15@yahoo.com

Received 22 May 2020; Accepted 18 June 2020; Published 16 July 2020

Guest Editor: Muhammad Javaid

Copyright (C) 2020 Ansheng Ye et al. This is an open access article distributed under the Creative Commons Attribution License, which permits unrestricted use, distribution, and reproduction in any medium, provided the original work is properly cited.

Topological index is a number that can be used to characterize the graph of a molecule. Topological indices describe the physical, chemical, and biological properties of a chemical structure. In this paper, we derive the analytical closed formulas of face index of some planar molecular structures such as $\mathrm{TUC}_{4}, \mathrm{TUC}_{4} C_{8}(S), \mathrm{TUHC}_{6}, \mathrm{TUC}_{4} C_{8}(R)$, and armchair $\mathrm{TUVC}_{6}$.

\section{Introduction}

In this time of rapid technological development, the pharmacological techniques have evolved rapidly during the recent years. Consequently, a large number of new drugs and chemical compounds have been obtained. A huge amount of work is required to study the biological, chemical, and pharmacological aspects of these new drugs and chemical compounds. This workload is becoming more and more cumbersome as it requires sufficient tools, reagents, human resources, and a lot of time to check the performance of these new chemical compounds. However, the developing countries cannot afford these equipment and reagents to check up these biochemical properties and are resultantly unable to compete with the developed world in the areas of medical science and industry. To some extent, the chemical graph theory solved this problem as it assists to measure the pharmaceutical, chemical, and physical properties of the chemical compounds. Fortunately, previous research has revealed that chemical properties of a molecule such as boiling point, melting point, and toxicity are closely related to their molecular structures (see $[1,2]$ ). This relationship is one of the key reasons for the development of the mathematical chemistry. In the chemical graph theory, a molecular structure can be represented in the form of a graph $G=(V(G), E(G))$, where vertices $V=V(G)$ and edges $E=$ $E(G)$ of a graph $G$ show the atoms and the bonds of a molecular structure, respectively.

A topological index (TI) is an invariant that is assigned to a molecular structure (graph) and is used to characterize the molecule. It may be thought as a convenient device which converts a chemical constitution into a unique number, which is independent of the way in which the corresponding graph has been drawn or labeled. TIs were employed in developing a suitable correlation between the chemical structure and chemical or biological activities and physical properties. Several researchers working in the area of chemical and mathematical sciences have introduced TIs, such as the Wiener index, Randić indices, Zagreb indices, PI index, eccentric index, atom-bond connectivity index, and forgotten index, which have been used to predict the characteristics of the nanomaterials, drugs, and other chemical compounds. There are several papers to calculate the topological indices of some special molecular graphs [3-12]. 
The notions of a planar graph, its faces, and an infinite face are well known in the literature. Let $G=(V(G), E(G), F(G))$ be a finite simple connected planar graph, where $V(G), E(G)$, and $F(G)$ represent the vertex, edge, and face sets, respectively. A face $f \in F(G)$ is incident to an edge $e \in E(G)$ if $e$ is one of those which surrounds the face. Similarly, a face $f \in F(G)$ is incident to a vertex $v$ in $G$ if $v$ is at the end of one of those incident edges; the incidency of $v$ to the face $f$ is represented by $v \sim f$. The face degree $f$ in $G$ is given as $d(f)=\sum_{v \sim f} d(v)$. For the notions and notations not given here, we refer [13] to the readers.

Recently, Jamil et al. [14] introduced a novel topological index named as the face index. The face index helped to predict the energy and the boiling points of selected benzenoid hydrocarbons with the correlation coefficient $r>0.99$. For a graph $G$, the face index $(\mathrm{FI})$ can be defined as

$$
\mathrm{FI}(G)=\sum_{f \in F(G)} d(f)=\sum_{v \sim f \in F(G)} d(v) .
$$

In this paper, we calculate the face index of some special molecular graphs which have been widely used in drugs.

\section{Main Results}

In this section, we investigate the exact formulas of the face index for the molecular structures of vastly studied nanotubes with wide range of applications: $\mathrm{TUC}_{4} C_{8}(S), \mathrm{TUC}_{4} C_{8}(R), \mathrm{TUHC}_{6}, \mathrm{TUC}_{4}$, and $\mathrm{TUVC}_{6}$. To find the face indices of the molecular graphs of these nanotubes, we partitioned the face set depending on the degrees of each face.

\subsection{Face Index of $T_{U} C_{4} C_{8}(S)[n, q, r]$ and $T U C_{4} C_{8}(R)$} $[n, q, r]$ Nanotubes. The 2-dimensional lattice of $\mathrm{TUC}_{4} \mathrm{C}_{8}(\mathrm{~S})$ $[n, q, r]$ is constructed by the alternatingly positioned squares $C_{4}$ and octagons $C_{8}$ (see Figure 1(a)), where $n, q$, and $r$ represent the number of rows, octagons in each row, and squares in each row. A $\mathrm{TUC}_{4} C_{8}(S)[n, q, r]$ nanotube can be constructed by rolling the $2 \mathrm{D}$ lattice of carbon atoms and can be seen in Figure 1(b).

Firstly, we prove the following formula which provides the exact values of the face index for $\operatorname{TUC}_{4} C_{8}(S)[n, q, r]$.

Theorem 1. Let $G=T U C_{4} C_{8}(S)[n, q, r]$, where $n, q, r \geq 1$, be the 2-dimensional lattice of $\mathrm{TUC}_{4} \mathrm{C}_{8}(S)$ nanotube; then, the face index of $G$ is given as

$$
\mathrm{FI}(G)=24 n q+12 n r+4 q+12 r .
$$

Proof. Let $G$ be the 2-dimensional lattice of $\mathrm{TUC}_{4} C_{8}(S)[n, q, r]$ nanotube with $n$ number of rows and let $q$ and $r$ be the number of octagons and number of squares in each row, respectively. In $\mathrm{TUC}_{4} C_{8}(S)[n, q, r]$, the total number of faces in one row is $q+r$. Let $f_{j}$ denote the faces having $\sum_{w \sim f_{j}} d_{w}=j$ and $\left|f_{j}\right|$ denote the number of faces with degree $j$. From Figure $1(\mathrm{a})$, it can be noticed that $2 \mathrm{D}$ lattice of $\mathrm{TUC}_{4} C_{8}(S)[7,4,4]$ contains four types of internal faces $f_{12}, f_{20}, f_{22}$, and $f_{24}$ and an external face, $f_{\infty}$. When
$\mathrm{TUC}_{4} C_{8}(S)[n, q, r]$ has $n$ rows, then sum of vertex degrees of external face is $8 q+12 r$. The number of internal faces in each row is given in Table 1 .

The face index of $\mathrm{TUC}_{4} C_{8}(S)[n, q, r]$ is

$$
\begin{aligned}
\mathrm{FI}(G) & =\sum_{w \sim f \in F(G)} d_{w} \\
& =\sum_{w \sim f_{12}} d_{w}+\sum_{w \sim f_{22}} d_{w}+\sum_{w \sim f_{24}} d_{w}+\sum_{w \sim f_{\infty}} d_{w} \\
& =\left|f_{12}\right|(12)+\left|f_{22}\right|(22)+\left|f_{24}\right|(24)+8 q+12 r \\
& =n r(12)+2 q(22)+q(n-2)(24)+8 q+12 r \\
& =12 n r+44 q+24 n q-48 q+8 q+12 r \\
& =24 n q+12 n r+4 q+12 r .
\end{aligned}
$$

This completes the proof.

Theorem 2. Let $n, q, r \geq 1$ and $H=T U C_{4} C_{8}(R)[n, q, r]$ be the graph of 2-dimensional lattice of $T U C_{4} C_{8}(R)$ nanotube. Then, the face index of the graph $H$ is given as

$$
\mathrm{FI}(H)=24 n q+6 n r+12 q+18 r .
$$

Proof. Consider a $\mathrm{TUC}_{4} C_{8}(R)[n, q, r]$ nanotube with $n$ number of rows, $q$ number of octagons, and $r$ number of squares in each row as shown in Figure 2(a). The 2-dimensional lattice $(H)$ of $\mathrm{TUC}_{4} C_{8}(R)[n, q, r]$ is shown in Figure 2(b). In $H$, the total number of faces in one row is $q+r$. Let $f_{j}$ and $\left|f_{j}\right|$ denote the face with degree $j$ and the number of faces with degree $j$, respectively. From the structure of $H$, one can notice that there are three types of internal faces $f_{11}, f_{12}$, and $f_{24}$ and an external face $f_{\infty}$. The external face has degree $12 q+2 r$. Table 2 illustrates the number of internal faces in $\mathrm{TUC}_{4} C_{8}(R)[n, q, r]$ based on the degree of each face.

The face index of the graph $H=\mathrm{TUC}_{4} C_{8}(R)[n, q, r]$ is

$$
\begin{aligned}
\mathrm{FI}(H) & =\sum_{w \sim f \in F(H)} d_{w} \\
& =\sum_{w \sim f_{11}} d_{w}+\sum_{w \sim f_{12}} d_{w}+\sum_{w \sim f_{24}} d_{w}+\sum_{w \sim f_{\infty}} d_{w} \\
& =\left|f_{11}\right|(11)+\left|f_{12}\right|(12)+\left|f_{24}\right|(24)+12 q+2 r \\
& =r(11)+\frac{(n-1) r}{2}(12)+n q(24)+12 q+2 r \\
& =11 r+6 n r-6 r+24 n q+12 q+2 r \\
& =24 n q+6 n r+12 q+7 r .
\end{aligned}
$$

This completes the proof.

2.2. Face Index of $T_{U} C_{4}[p, q]$ Nanotube. For $p \geq 2$ and $q \geq 3$, the 2-dimensional lattice of $\operatorname{TUC}_{4}[p, q]$ nanotube is obtained by the Cartesian product of the path $P_{p}$ and the cycle $C_{q}$. For $p=8$ and $q=4$, the example of $\operatorname{TUC}_{4}[p, q]$ is shown in Figure 3(b). 


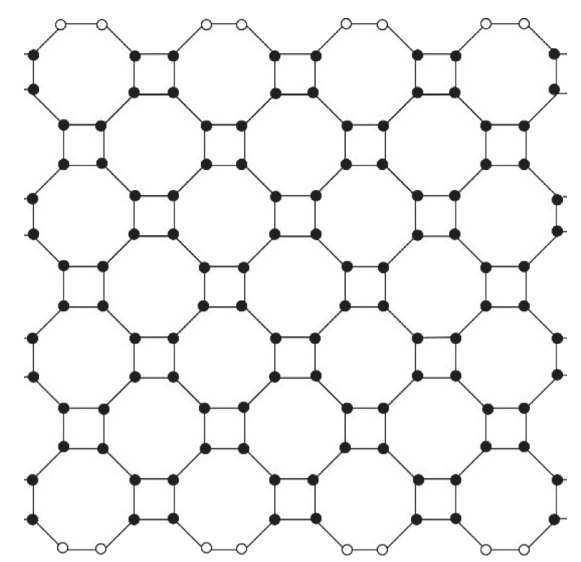

(a)

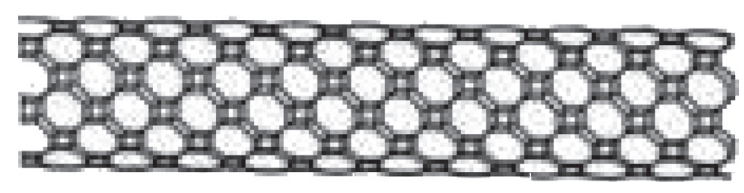

(b)

Figure 1: (a) 2D lattice of $\mathrm{TUC}_{4} C_{8}(S)[7,4,4]$. (b) $3 \mathrm{D}$ nanotube $\mathrm{TUC}_{4} C_{8}(S)$.

TABLE 1: Numbers of $f_{12}, f_{20}, f_{22}$, and $f_{24}$ with given number of rows.

\begin{tabular}{|c|c|c|c|c|}
\hline$n$ & $\left|f_{12}\right|$ & $\left|f_{20}\right|$ & $\left|f_{22}\right|$ & $\left|f_{24}\right|$ \\
\hline 1 & $R$ & $q$ & - & - \\
\hline 2 & $2 r$ & - & $2 q$ & - \\
\hline 3 & $3 r$ & - & $2 q$ & Q \\
\hline 4 & $4 r$ & - & $2 q$ & $2 q$ \\
\hline . & . & . & . & . \\
\hline$\cdot$ & . & . & . & . \\
\hline$\cdot$ & . & . & . & . \\
\hline $\mathrm{N}$ & $\mathrm{Nr}$ & - & $2 q$ & $(n-2) q$ \\
\hline
\end{tabular}

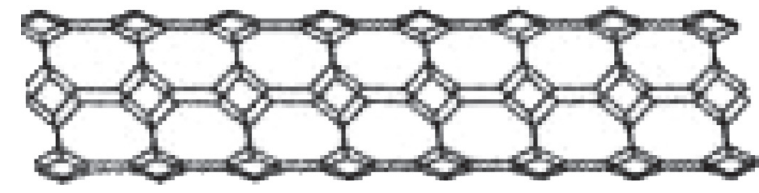

(a)

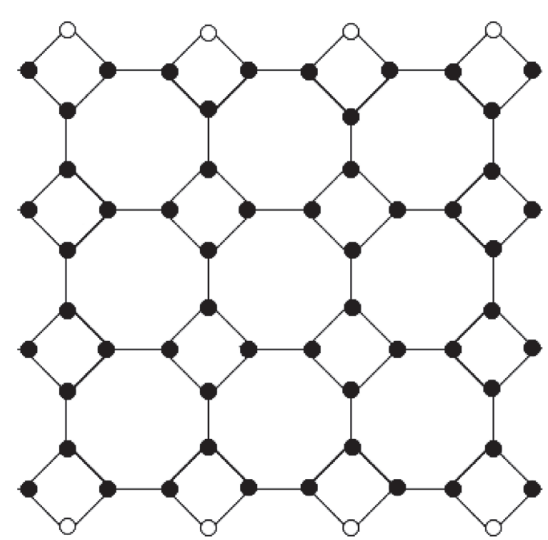

(b)

Figure 2: (a) 3D nanotube $\mathrm{TUC}_{4} C_{8}(R)$. (b) $2 \mathrm{D}$ lattice of $\mathrm{TUC}_{4} C_{8}(R)$.

TABLE 2: Numbers of $f_{11}, f_{12}$, and $f_{24}$ with given number of rows.

\begin{tabular}{lccc}
\hline$n$ & $\left|f_{11}\right|$ & $\left|f_{12}\right|$ & $\left|f_{24}\right|$ \\
\hline 1 & $R$ & - & $Q$ \\
2 & $R$ & $r / 2$ & $2 q$ \\
3 & $R$ & $\mathrm{R}$ & $3 q$ \\
4 & $R$ & $3 r / 2$ & $4 q$ \\
5 & 5 & $2 r$ & $5 q$ \\
$\cdot$ & $\cdot$ & $\cdot$ & $\cdot$ \\
$\cdot$ & $\cdot$ & $\cdot$ & $\cdot$ \\
. & $\cdot$ & $(n-1) r / 2$ &. \\
$N$ & $R$ & & $N q$ \\
\hline
\end{tabular}




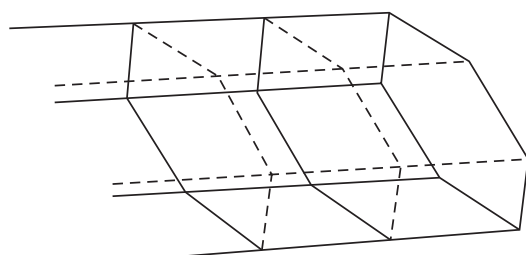

(a)

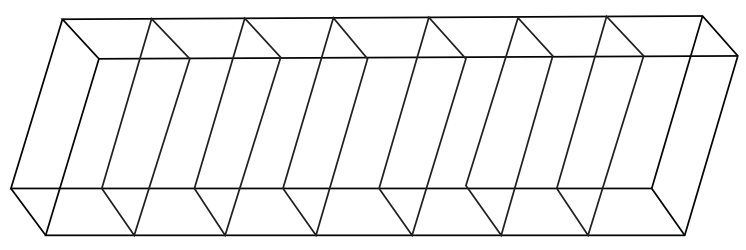

(b)

Figure 3: (a) Nanotube $\operatorname{TUC}_{4}[p, 6]$. (b) Nanotube $\operatorname{TUC}_{4}[8,4]$.

Theorem 3. Let $K$ be the graph of $T_{U C}[p, q]$ nanotube structure, where $p \geq 2$ and $q \geq 3$. Then, the face index of $K$ is equal to

$$
\mathrm{FI}(K)= \begin{cases}18 q ; & \text { if } p=2, \\ 34 q ; & \text { if } p=3, \\ 2 q(8 p-7) ; & \text { if } p>3 .\end{cases}
$$

Proof. We will prove the result for $p>3$. Let $K$ denote the graph of $\mathrm{TUC}_{4} C[p, q]$ nanotube structure. From Figure 3, we can notice that the graph $K$ contains three types of internal faces, namely, $f_{3 q}, f_{14}$, and $f_{16}$, and an external face of degree $3 q$. By applying the definition and using the values from Table 3, the face index of $K$ can be computed as

$$
\begin{aligned}
\operatorname{FI}(K) & =\sum_{w \sim f \in F(K)} d_{w} \\
& =\sum_{w \sim f_{3 q}} d_{w}+\sum_{w \sim f_{14}} d_{w}+\sum_{w \sim f_{16}} d_{w} \\
& =\left|f_{3 q}\right|(3 q)+\left|f_{14}\right|(14)+\left|f_{16}\right|(16) \\
& =2(3 q)+14(2 q)+16(p-3) q \\
& =2 q(8 p-7) .
\end{aligned}
$$

This completes the proof.

2.3. Zig-Zag TUHC ${ }_{6}[n, q]$ Nanotube. Consider the graph $K$ of $\mathrm{TUHC}_{6}[n, q]$ zig-zag polyhex nanotube structure, where $n$ denotes the number of rows and the number of hexagons in each row is represented by $q$. Figure 4 illustrates the nanotube $\mathrm{TUHC}_{6}[n, q]$ and its 2-dimensional structure.

Theorem 4. For $n, q \geq 1$, let $K$ represent the 2-dimensional graph of $\mathrm{TUHC}_{4}[n, q]$ structure. The face index of $K$ is

$$
\operatorname{FI}(K)=18 n q+8 q \text {. }
$$

Proof. Let $\mathrm{TUHC}_{6}[n, q]$ be a polyhex nanotube with $n$ number of rows and $q$ number of hexagons in each row and $K$ be the 2-dimensional graph of $\mathrm{TUHC}_{6}[n, q]$ structure. The molecular graph of $\mathrm{TUHC}_{6}[n, q]$ is shown in Figure 4. Let $f_{j}$ denote the face having degree $j$, i.e., $\sum_{w \sim f} d_{w}=j$, and let $\left|f_{j}\right|$ denote the number of $f_{j}$. The molecular graph of TUHC $_{6}[n, q]$ contains two types of internal faces $f_{17}$ and $f_{18}$ and an external face $f_{\infty}$. When $\operatorname{TUHC}_{6}[n, q]$ has $n$ rows, then the face degree of $f_{\infty}$ is $10 q$. The number of internal faces with the given number of rows is listed in Table 4 .
TABLE 3: Numbers of $f_{11}, f_{12}$, and $f_{24}$ with given number of rows.

\begin{tabular}{lcccc}
\hline TUC $_{4}[k, q]$ & $\left|f_{3 q}\right|$ & $\left|f_{12}\right|$ & $\left|f_{14}\right|$ & $f_{16}$ \\
\hline$k=2$ & 2 & $\mathrm{Q}$ & - & - \\
$k=3$ & 2 & - & $2 q$ & - \\
$k=4$ & 2 & - & $2 q$ & $\mathrm{Q}$ \\
$k=5$ & & - & $2 q$ & $2 q$ \\
$\cdot$ & $\cdot$ & $\cdot$ & $\cdot$ & $\cdot$ \\
$\cdot$ & $\cdot$ & $\cdot$ & $\cdot$ & $\cdot$ \\
$\cdot$ & $\cdot$ & $\cdot$ & $\cdot$ & $\cdot$ \\
$k=p$ & 2 & - & $2 q$ & $(p-3) q$ \\
\hline
\end{tabular}

The face index of the graph $K=\mathrm{TUHC}_{6}[n, q]$ is

$$
\begin{aligned}
\mathrm{FI}(K) & =\sum_{w \sim f \in F(K)} d_{w} \\
& =\sum_{w \sim f_{17}} d_{w}+\sum_{w \sim f_{18}} d_{w}+\sum_{w \sim f_{\infty}} d_{w} \\
& =\left|f_{17}\right|(17)+\left|f_{18}\right|(18)+10 q \\
& =2 q(17)+(n-2) q(18)+10 q \\
& =34 q+18 n q-36 q+10 q \\
& =18 n q+8 q,
\end{aligned}
$$

which is the required result.

2.4. TUVC $6[n, q]$ Nanotube. The 2-dimensional graph of $\operatorname{TUVC}_{6}[n, q]$ is shown in Figure 5(b), and the $\operatorname{TUVC}_{6}[n, q]$ nanotube can be constructed by rolling this lattice of carbon atoms (Figure 5(a)), where $n$ represents the number of rows and $q$ is the hexagons in each row.

Theorem 5. Let $L=\operatorname{TUVC}_{4}[n, q]$, where $n, q \geq 1$, be the graph of 2-dimensional lattice of $\mathrm{TUVC}_{6}[n, q]$ armchair polyhex nanotube. The face index of $L$ is

$$
\mathrm{FI}(L)=36 n q-8 q \text {. }
$$

Proof. Let $L$ represent the 2-dimensional molecular graph of $\mathrm{TUVC}_{6}[n, q]$ with $n$ number of rows and $q$ number of hexagons in each row. From Figure 5(b), we can easily notice that $L$ contains 2 types of internal faces $f_{16}$ and $f_{18}$ and an external face, and the degree of external face is $14 q$. The cardinalities of internal faces with given degree and given number of rows are explained in Table 5.

The face index of $L=\operatorname{TUVC}_{6}[n, q]$ is 

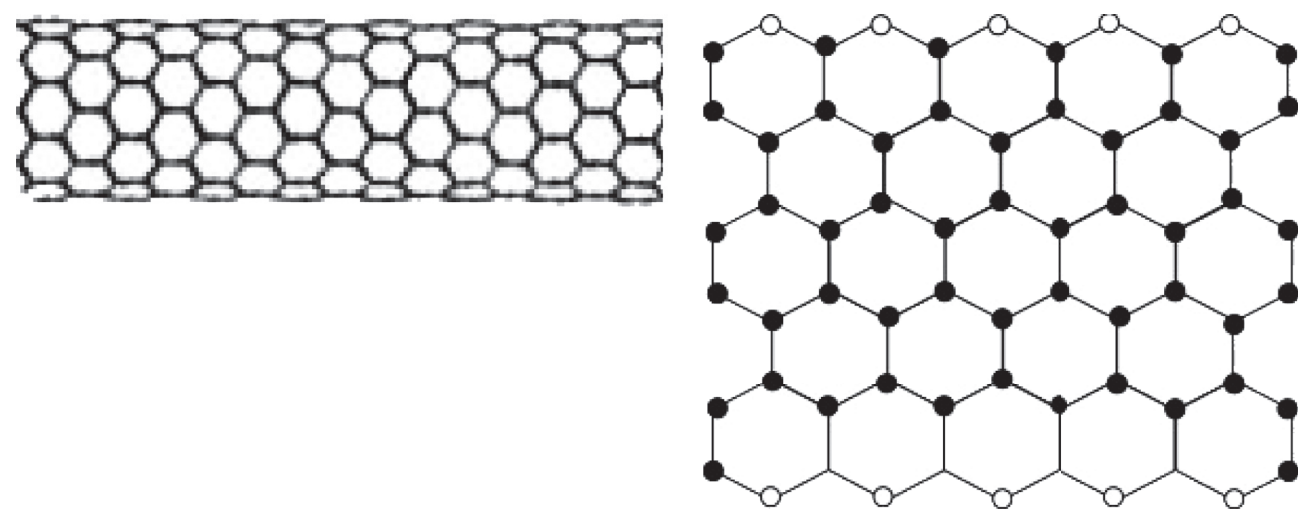

(a)

(b)

Figure 4: (a) 3D nanotube $\mathrm{TUHC}_{6}[n, q]$. (b) 2D lattice of $\mathrm{TUHC}_{6}[5,5]$.

TABLE 4: The cardinalities of the faces with given degree for given number of rows.

\begin{tabular}{lccc}
\hline$n$ & $\left|f_{16}\right|$ & $\left|f_{17}\right|$ & \\
\hline 1 & $q$ & - & - \\
2 & - & $2 q$ & - \\
3 & - & $2 q$ & $\mathrm{Q}$ \\
4 & - & $2 q$ & $\cdot f_{18} \mid$ \\
$\cdot$ & $\cdot$ & $\cdot$ & $\cdot$ \\
$\cdot$ & $\cdot$ & $\cdot$ & $\cdot$ \\
$\cdot$ & $\cdot$ & $2 q$ & $\cdot$ \\
\hline
\end{tabular}

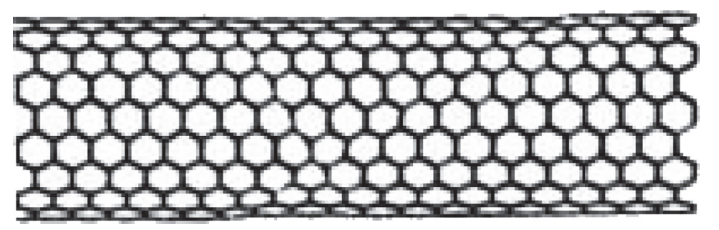

(a)

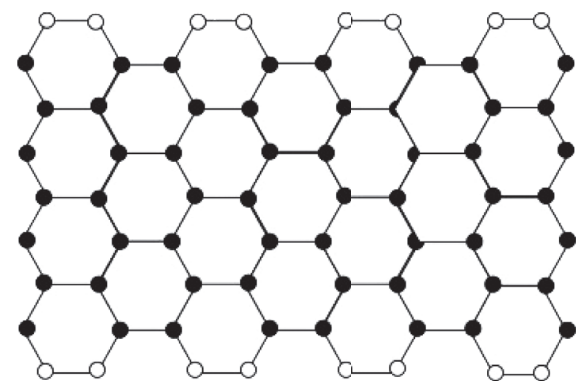

(b)

FIgURe 5: (a) Armchair 3D nanotube $\operatorname{TUVC}_{6}[n, q]$. (b) 2D lattice of $\mathrm{TUVC}_{6}[4,4]$.

TABLE 5: The cardinalities of $f_{14}, f_{16}$, and $f_{18}$ with given number of rows.

\begin{tabular}{cccc}
\hline$n$ & $\left|f_{14}\right|$ & $\left|f_{16}\right|$ & $\left|f_{18}\right|$ \\
\hline 1 & $q$ & - & - \\
2 & - & $2 q$ & $\mathrm{Q}$ \\
3 & - & $2 q$ & $3 q$ \\
4 & - & $2 q$ & $5 q$ \\
$\cdot$ & $\cdot$ & $\cdot$ & $\cdot$ \\
$\cdot$ & $\cdot$ & $\cdot$ & $\cdot$ \\
$\cdot$ & $\cdot$ & $\cdot$ & $\cdot$ \\
$N$ & - & $2 q$ & $(2 n-3) q$ \\
\hline
\end{tabular}

$$
\begin{aligned}
\mathrm{FI}(L) & =\sum_{w \sim f \in F(L)} d_{w} \\
& =\sum_{w \sim f_{16}} d_{w}+\sum_{w \sim f_{18}} d_{w}+\sum_{w \sim f_{\infty}} d_{w} \\
& =\left|f_{16}\right|(16)+\left|f_{18}\right|(18)+14 q \\
& =2 q(16)+(2 n-3) q(18)+14 q \\
& =32 q+36 n q-54 q+14 q \\
& =36 n q-8 q,
\end{aligned}
$$

and the proof is complete. 


\section{Conclusion}

In [14], using multiple linear regression, it has been shown that the novel face index can predict the $\pi$ electron energy and boiling point of benzenoid hydrocarbon with a correlation coefficient greater than 0.99 . Therefore, this index can be useful in QSPR/QSAR studies. In this paper, we have computed the novel face index of some nanotubes.

\section{Data Availability}

No data were used to support the study.

\section{Disclosure}

This research was carried out as a part of the employment of the authors.

\section{Conflicts of Interest}

The authors declare that they have no conflicts of interest.

\section{References}

[1] H. Wiener, "Structural determination of paraffin boiling points," Journal of the American Chemical Society, vol. 69, no. 1, pp. 17-20, 1947.

[2] A. R. Katritzky, R. Jain, A. Lomaka, R. Petrukhin, U. Maran, and M. Karelson, "Perspective on the relationship between melting points and chemical structure," Crystal Growth \& Design, vol. 1, no. 4, pp. 261-265, 2001.

[3] J.-B. Liu, M. Javaid, and H. M. Awais, "Computing Zagreb indices of the subdivision-related generalized operations of graphs," IEEE Access, vol. 7, pp. 105479-105488, 2019.

[4] J.-B. Liu, S. Javed, M. Javaid, and K. Shabbir, "Computing first general Zagreb index of operations on graphs," IEEE Access, vol. 7, pp. 47494-47502, 2019.

[5] J.-H. Tang, U. Ali, M. Javaid, and K. Shabbir, "Zagreb connection indices of subdivision and semi-total point operations on graphs," Journal of Chemistry, vol. 2019, Article ID 9846913, 14 pages, 2019.

[6] M. Javaid, M. U. Rehman, and J. Cao, "Topological indices of rhombus type silicate and oxide networks," Canadian Journal of Chemistry, vol. 95, no. 2, pp. 134-143, 2017.

[7] A. Aslam, Y. Bashir, S. Ahmad, and W. Gao, "On topological indices of certain dendrimer structures," Zeitschrift für Naturforschung A, vol. 72, no. 6, pp. 559-566, 2017.

[8] A. Aslam, S. Ahmad, and W. Gao, "On certain topological indices of boron triangular nanotubes," Zeitschrift für Naturforschung A, vol. 72, no. 8, pp. 711-716, 2017.

[9] A. Aslam, M. K. Jamil, W. Gao, and W. Nazeer, "Topological aspects of some dendrimer structures," Nanotechnology Reviews, vol. 7, no. 2, pp. 123-129, 2018.

[10] A. Aslam, S. Ahmad, M. A. Binyamin, and W. Gao, "Calculating topological indices of certain OTIS interconnection networks," Open Chemistry, vol. 17, no. 1, pp. 220-228, 2019.

[11] Z. Shao, P. Wu, Y. Gao, I. Gutman, and X. Zhang, "On the maximum $\mathrm{ABC}$ index of graphs without pendent vertices," Applied Mathematics and Computation, vol. 315, pp. 298-312, 2017.

[12] Z. Shao, P. Wu, X. Zhang, D. Dimitrov, and J.-B. Liu, "On the maximum $\mathrm{ABC}$ index of graphs with prescribed size and without pendent vertices," IEEE Access, vol. 6, no. 6, pp. 27604-27616, 2018.

[13] J. A. Bondy and U. S. R. Murty, Graph Theory, Springer, Berlin, Germany, 2008.

[14] M. K. Jamil, M. Imran, and K. Abdul Sattar, "Novel face index for benzenoid hydrocarbons," Mathematics, vol. 8, no. 3, p. $312,2020$. 